\title{
Auto-localization algorithm for local positioning systems
}

\author{
J. Guevara*, A.R. Jiménez, J.C. Prieto, F. Seco
}

\begin{abstract}
A B S T R A C T
This paper studies the problem of determining the position of beacon nodes in Local Positioning Systems (LPSs), for which there are no inter-beacon distance measurements available and neither the mobile node nor any of the stationary nodes have positioning or odometry information. The common solution is implemented using a mobile node capable of measuring its distance to the stationary beacon nodes within a sensing radius. Many authors have implemented heuristic methods based on optimization algorithms to solve the problem. However, such methods require a good initial estimation of the node positions in order to find the correct solution. In this paper we present a new method to calculate the inter-beacon distances, and hence the beacons positions, based in the linearization of the trilateration equations into a closed-form solution which does not require any approximate initial estimation. The simulations and field evaluations show a good estimation of the beacon node positions.
\end{abstract}

\section{Introduction}

There are many location-aware applications, such as object position tracking in smart spaces, and personal navigation, that require systems for indoor localization. The Global Positioning System (GPS) is not available indoors, therefore new localization technologies are required. Localization systems designed to work in indoor environments are known as Local Positioning Systems (LPSs) [1]. These systems require the installation of several nodes at fixed positions (called beacon nodes) in the indoor environment. Beacon nodes are usually positioned at the ceiling or on walls, and a mobile node is attached to the person or object to locate. In order to locate the mobile node using the trilateration method the position of the beacons must be known in advance. The determination of the beacons position is usually done manually by measuring the distance to the two closest walls of the building using measuring tapes or ultrasonic/laser rangers. This method is cumbersome and error prone, therefore different techniques have been proposed to address the problem of obtaining automatically the position of the beacons, also known as the autocalibration or auto-localization problem.

Typical auto-localization solutions are based on measuring distances from a group of localized nodes, or a mobile node at several known positions, to the beacons with unknown position and then performing a inverse trilateration (locating the beacons using the mobile node). This method of inverse positioning states that with enough measurements all the beacons can be localized by trilateration techniques [2]. In [3] four different mobile node positions are used to locate the beacon nodes of a 3D LPS. In [4] three nodes with known positions are required plus a group of nodes with unknown positions. In [5] only the relative distances between four nodes mounted on a mobile robot are required. These methods, however, require an external localization system to obtain the position of the mobile node at each static location, which is not always available in indoor environments.

A more generic problem is addressed by assuming that no information of the position of any node is known. In 
these cases the only available data are the distance measurements between the beacons and the mobile node. Duff and Muller [6] used a nonlinear least-square optimization algorithm to locate the beacons, where the objective functions were the distance equations and the variables were the coordinates of all nodes. Since a first position estimation of all nodes is required, as initial conditions for the algorithm, a trial and error method was used by randomly generating those conditions and choosing the best solution.

In $[7,8]$ an Extended Kalman Filter and an $\mathrm{H}$-infinite filter are used respectively to obtain the position of the beacons and the mobile node. In both cases an approximate initial position estimation of the beacons is obtained using dead-reckoning information of the mobile node (positioned on a mobile vehicle) and measured node to beacon ranges. In [9], a distance matrix is formed with the range measurements between beacons and the mobile node at different locations. With that information a rough approximation of the inter-beacon ranges is obtained using an interpolation scheme. However, these initial distance estimates are not precise enough to be used to locate the beacons. Therefore, a heuristic optimization method that uses a non-linear objective function is previously carried out.

In the present paper we propose a new closed-form solution for the position estimation of the beacons that neither requires a trial and error approximation (i.e., randomly generated positions) nor any external positioning information (such as dead-reckoning data). Section 2 describes the auto-localization problem when no position information of any node is known. In Section 3 a new method to locate three beacon nodes without initial estimations is presented. In Section 4 the method is expanded for any number of beacons. The proposed solution is then evaluated by simulation in Section 5 , and also experimentally on an ultrasonic 3D LPS system in Section 6.

\section{The auto-localization problem}

Fig. 1 shows a typical configuration of a LPS, composed by a group of four static nodes $N_{i}, i=1, \ldots, 4$, called beacon

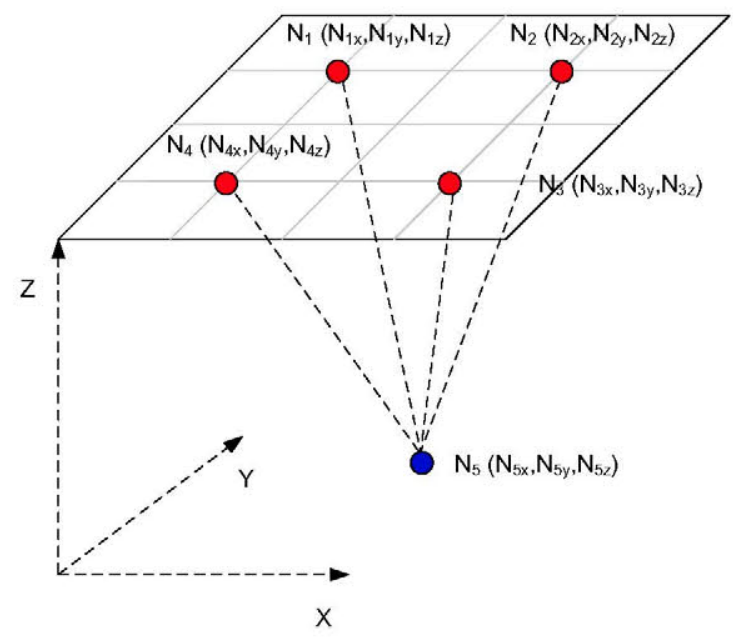

Fig. 1. 3D local positioning system. nodes, and one mobile node $N_{5}$. If all the beacons' positions are known the mobile node coordinates $\left(N_{5 x}, N_{5 y}, N_{5 z}\right)$ can be calculated using the distance equations, known as trilateration or multilateration equations:

$\left\|\mathbf{N}_{\mathbf{5}}-\mathbf{N}_{\mathbf{1}}\right\|=d_{15}$

$\left\|\mathbf{N}_{\mathbf{5}}-\mathbf{N}_{\mathbf{2}}\right\|=d_{25}$,

$\left\|\mathbf{N}_{\mathbf{5}}-\mathbf{N}_{\mathbf{4}}\right\|=d_{45}$

where $d_{i 5}, i=1, \ldots, 4$ is the distance between the beacon $N_{i}$ and the mobile node $N_{5}$.

The objective of auto-localization algorithms is to obtain the position of all nodes, beacons and mobile, using only a group of distance measurements between the beacons and the mobile node. The distance measurements are obtained at different unknown positions of the mobile node. In most LPS inter-beacon distances are not available since they are only designed to measure their distance to the mobile node. Besides, no external localization system is used, so no positioning or odometric information is available in any node.

The typical approach used for auto-localization is to formulate it as an optimization problem, where the objective function to be minimized includes the residuals of the distance Eq. (1) and the variables in the search space are the coordinates of all the nodes. In principle, the problem is solvable by obtaining more distance measurements than the number of unknown variables. However, such direct approach, known as degrees of freedom analysis, does not guarantee a unique solution in a system of nonlinear equations, such as the trilateration equations. Priyantha et al. [10] introduced the concept of rigidity in LPS systems to develop a method that ensures a unique solution of the auto-localization problem. The process requires a starting subset of nodes (a starting group of beacons and mobile nodes) that have been verified to have a unique solution. Then, an incremental process of adding more nodes, based in the rigidity theory, is used to preserve the uniqueness of the solution. Priyantha also developed a group of movement strategies to obtain the starting subset of nodes, but these strategies were only justified using a degrees of freedom analysis. In [11] the precise conditions for obtaining different initial subsets of nodes with a unique solution were presented and justified using rigidity theory.

Another problem presented with the optimization algorithms, and also with Bayesian methods, is that their convergence depend heavily on the initial conditions used. Here we propose a new method based in the linearization of the trilateration equations by expanding these equations and grouping all nonlinear terms in additional variables, therefore circumventing the convergence problem.

\section{Linear solution of the auto-localization problem for three beacons}

The linearization of the trilateration equations is not an easy task. The equations are nonlinear and the only data available are the distances between the beacons and the mobile node. Therefore, in order to obtain a linear set of 
equations, it will be convenient to restrain some of the conditions of the auto-localization problem. In the present paper the case where all the beacons nodes are located in a plane parallel to the plane containing the mobile node trajectory is considered. Such configuration is very common in LPS systems, for example when all the beacons are located on the ceiling, and the mobile node movement is made at an approximately constant height from the floor. For this particular case the conditions for a solvable initial subset of three beacon nodes are obtained based in [11], where at least four nonaligned mobile positions are required.

\subsection{Linearization of the trilateration equations for three beacons}

Fig. 2 shows the chosen initial subset composed of the beacon nodes $N_{i}, i=1,2,3$ and the four measurement points $N_{i}, i=4, \ldots, 7$ from the mobile node path. From now on, the measurement points of the mobile node will be referred as virtual nodes. Without loss of generality, a coordinate system can be defined using the beacon nodes where the coordinates of $N_{1}$ are $(0,0,0), N_{2}$ are $\left(N_{2 x}, 0,0\right)$ and $N_{3}$ are $\left(N_{3 x}, N_{3 y}, 0\right)$. Then, the trilateration equations can be rewritten as a function of two groups of distance measurements: the inter-beacon distances $d_{12}, d_{13}, d_{23}$ and the volume of the tetrahedron $V_{t}$ (formed by the beacons and any virtual node) which are unknown variables, and the distances between beacon nodes and virtual nodes $d_{14}, d_{24}, d_{34}, \ldots$, which are the available data. Appendix A shows that the trilateration equations can be expressed in the linear form $A X=B$ where:

$$
\begin{aligned}
A & =\left[\begin{array}{cccccc}
d_{34}^{2} & d_{24}^{2} & d_{14}^{2} & D_{324} D_{214} & -D_{314} D_{214} & -1 \\
d_{35}^{2} & d_{25}^{2} & d_{15}^{2} & D_{325} D_{215} & -D_{315} D_{215} & -1 \\
\vdots & \vdots & \vdots & \vdots & \vdots & \vdots \\
d_{39}^{2} & d_{29}^{2} & d_{19}^{2} & D_{329} D_{219} & -D_{319} D_{219} & -1
\end{array}\right], \\
D_{i j k} & =d_{i k}^{2}-d_{j k}^{2}, \quad i, j=1,2,3 \quad k=4,5, \ldots, 9
\end{aligned}
$$

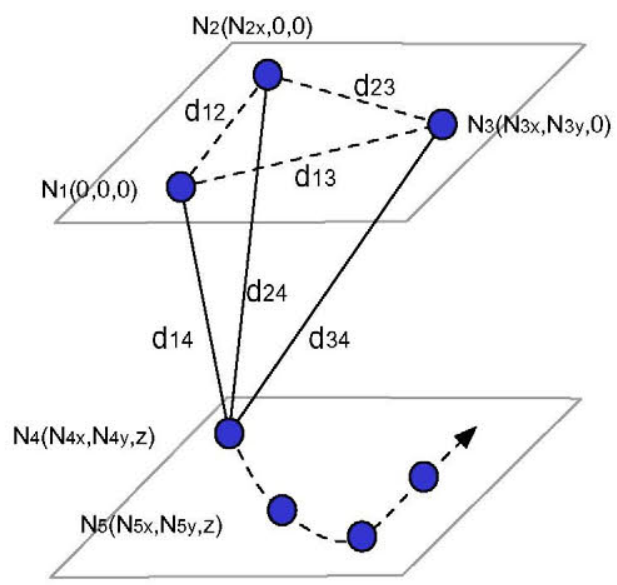

Fig. 2. Solvable node subset composed by three beacon nodes and four virtual nodes on a plane.

$$
X=\frac{1}{d_{12}^{2}}\left[\begin{array}{c}
d_{12}^{2}\left(d_{13}^{2}+d_{23}^{2}-d_{12}^{2}\right) \\
d_{13}^{2}\left(d_{12}^{2}+d_{23}^{2}-d_{13}^{2}\right) \\
d_{23}^{2}\left(d_{12}^{2}+d_{13}^{2}-d_{23}^{2}\right) \\
d_{13}^{2} \\
d_{23}^{2} \\
144 V_{t}^{2}+d_{12}^{2} d_{13}^{2} d_{23}^{2}
\end{array}\right],
$$

$B=\left[\begin{array}{c}D_{314} D_{324} \\ D_{315} D_{325} \\ \vdots \\ D_{319} D_{329}\end{array}\right]$

The matrix $A$ and the vector $B$ are composed of the distance measurements from the virtual nodes. The vector $X$ includes the unknown inter-beacon distances. Since the linearization process added more variables, six instead of four virtual nodes are required to solve the equations. Once $X$ is obtained the unknown variables $d_{12}, d_{13}, d_{23}$ can be calculated with:

$d_{12}=\frac{1}{\sqrt{2}} \sqrt{\frac{X_{2}}{X_{4}}+\frac{X_{3}}{X_{5}}}$,
$d_{13}=\frac{1}{\sqrt{2}} \sqrt{X_{1}+\frac{X_{3}}{X_{5}}}$,
$d_{23}=\frac{1}{\sqrt{2}} \sqrt{X_{1}+\frac{X_{2}}{X_{4}}}$.

\section{2. $2 \mathrm{D}$ beacon localization for three beacons}

Once the inter-beacon distances are determined, the beacon nodes coordinates can be calculated using A.6, A.10 and A.11 from Appendix A:

$N_{1}=(0,0)$,

$N_{2}=\left(d_{12}, 0\right)$,

$N_{3}=\left(\frac{d_{12}^{2}+d_{13}^{2}-d_{23}^{2}}{2 d_{12}}, \pm \sqrt{d_{13}^{2}-\left(\frac{d_{12}^{2}+d_{13}^{2}-d_{23}^{2}}{2 d_{12}}\right)^{2}}\right)$.

The global coordinate system is defined so that the $Y$ coordinate of the third beacon is always positive. With the proposed equations an estimated position of the initial subset of beacon nodes can be obtained based only on range measurements. Before expanding the problem to more beacons we will evaluate the error propagated from the distance measurements in the linearized equations.

\subsection{Sensitivity analysis of the linearized equations}

The solution of the linearized equations is affected by the errors in the measured distances used in the matrices $A$ and $B$. The magnitude and type of error depends on the technol- 
ogy used in the LPS system. The algebraical manipulation applied to the trilateration equations further increases the complexity of the error propagation analysis. Consider the case where the distance measurements are given by

$d_{i j}=\overline{d_{i j}}+\epsilon$,

where $\overline{d_{i j}}$ is the true distance between node $i$ and $j$, and $\epsilon$ is the measurement error modeled as a zero mean Gaussian distribution with variance $\sigma^{2}$. Since matrices $A$ and $B$ are composed by the squared measured distances, the resultant error distribution is not Gaussian, though it can be modelled as such using a first order Taylor series expansion [12]:

$\operatorname{var}\left(d_{i j}^{2}\right) \approx 4 \overline{d_{i j}^{2}} \sigma^{2}$.

Therefore, the errors, or perturbations, present on matrices $A$ and $B$ are expected to increase with the distance between measured nodes. Overall, we can obtain an upper bound of the error $\delta X$ originated from the perturbation in the matrices $A$ and $B$ based on the condition number $\kappa(A)=\|A\|\left\|A^{-1}\right\|[13]$. By considering that the perturbation is small compared to the elements of matrix $A$ so $\left\|A^{-1}\right\|\|\delta A\|<1$ we have that:

$\frac{\|\delta X\|}{\|X\|} \leqslant \kappa(A)\left(\frac{\|\delta A\|}{\|A\|}+\frac{\|\delta B\|}{\|B\|}\right)$,

where $\|\cdot\|$ represents the matrix norm.

Although the condition number overestimates the magnitude of the solution's error, since (10) is an upper bound, a large condition number still shows a greater sensitivity to the errors on matrices $A$ and $B$. Therefore, a condition number of $10^{4}$ is used in our proposed algorithm in order to avoid ill-conditioned solutions. This value is used to validate a given solution obtained by the linearized equations, any solution with a higher condition number is discarded. Notice that the condition number does not provide a direct upper bound on the estimated inter-beacon distances error, but rather on the variables defined on (4). The inter-beacon distances error is related with the condition number but not proportionally (e.g., a condition number of 10 does not mean that the inter-beacon distances will have up to ten times the error of the range measurements). Therefore, the maximum value of the condition number was chosen by tests.

In our tests we found that the condition number of the linearized equations can be reduced by subtracting all equations by the last one. This procedure is based on the centering method which is used to improve the condition number of a given matrix. In this method the matrix $A$ is reduced by dropping the column of ones and subtracting the mean (or other typical value) from the remaining columns [18]. We can achieve this by locating the last virtual node near the center of the mobile node path in order to subtract the mean of the columns. The final auto-localization Eqs. (11)-(13) are expressed as:

$$
X=\frac{1}{d_{12}^{2}}\left[\begin{array}{c}
d_{12}^{2}\left(d_{13}^{2}+d_{23}^{2}-d_{12}^{2}\right) \\
d_{13}^{2}\left(d_{12}^{2}+d_{23}^{2}-d_{13}^{2}\right) \\
d_{23}^{2}\left(d_{12}^{2}+d_{13}^{2}-d_{23}^{2}\right) \\
d_{13}^{2} \\
d_{23}^{2}
\end{array}\right],
$$

$$
B=\left[\begin{array}{c}
D_{314} D_{324}-D_{319} D_{329} \\
D_{315} D_{325}-D_{319} D_{329} \\
\vdots \\
D_{318} D_{328}-D_{319} D_{329}
\end{array}\right]
$$

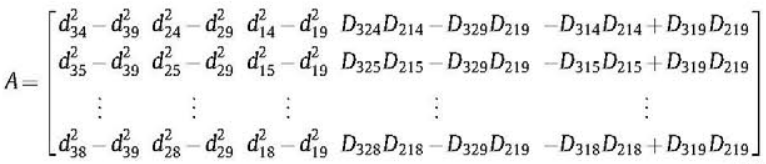

where $D_{i j k}$ is defined in (3). The solution of the inter-beacon distances $d_{12} d_{13}$ and $d_{23}$ are the same specified in (6).

\section{Auto-localization algorithm for more than three beacons}

In this section the auto-localization problem when there are more than three beacon nodes is considered. Our approach is very straightforward, we move the mobile node in order to obtain as many inter-beacon distances as possible, using the equations proposed in Section 3. Since it is assumed that the beacons lie on a plane, their positions can be solved using a 2D localization algorithm. A flowchart of the proposed algorithm is shown on Fig. 3.

\subsection{Calculation of multiple inter-beacon distances}

First the mobile node is moved on a plane trying to obtain at least $m$ measurement distances shared by the beacon nodes where, based in (13) and (11), $m$ is at least six, although adding redundant measurements improves the estimation. The path chosen in this paper is a circular route plus a measurement point at its center. The circular route was chosen to avoid aligned points and the point at its center to improve the condition number when applying the centering method of Eqs. (11)-(13). On large areas, or if the nodes' range is limited, multiple paths can be required. The goal is to obtain all possible inter-beacon distances applying the linearized equations on subsets of three beacons. Every subset is tested to confirm it shares distance measurements with $m$ virtual nodes or more. If so, the respective inter-beacon distances are calculated. To validate the obtained distances, the condition number of the matrix $A$ is verified to be less than our defined threshold of $10^{4}$.

Once the inter-beacon distances are validated, another three beacon subset is chosen. The process is repeated until every possible subset is evaluated. Since in the process a given pair of beacons is chosen more than once, multiple distance estimations for each pair of beacons can be obtained. In these cases the mean of all distance estimations is used. Once all groups of three beacons are evaluated, a 2D localization algorithm is used to locate every beacon position.

\section{2. $2 D$ beacon localization for more than three beacons}

There are several localization methods based on range measurements that can be employed to determine the beacons' position, such as the iterative trilateration. This method requires a group of anchor nodes with known 


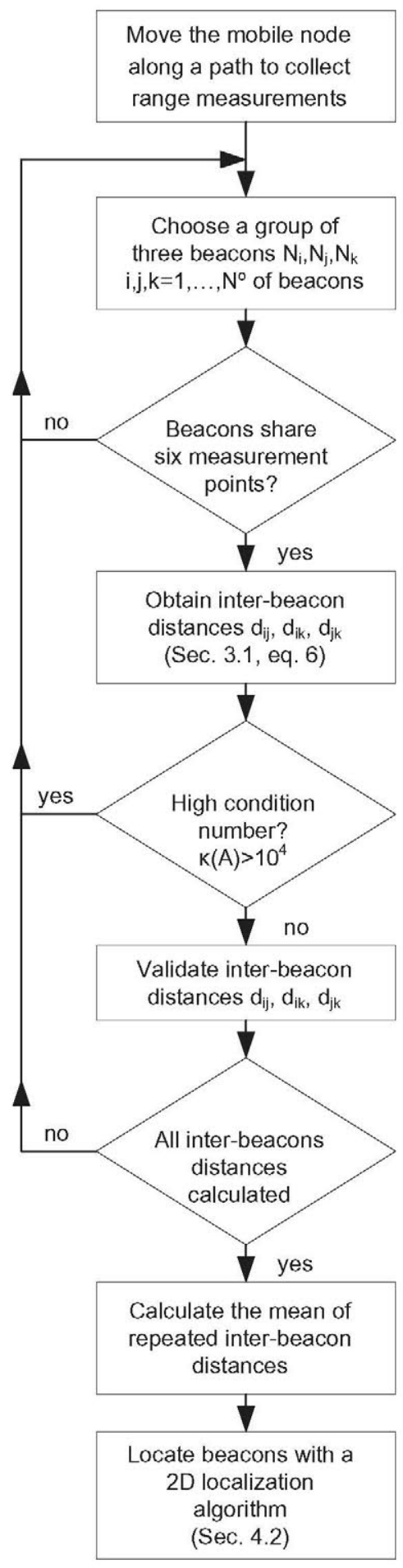

Fig. 3. Linearized auto-localization algorithm for more than three beacons.

positions which are used to locate other beacons within range. Later the new located beacons are used as anchors to locate more beacons. However, as the number of beacons increases the accumulated error in the beacons' position estimation increases [14]. A method to compute all beacons' positions simultaneously is the MDS-MAP which is based in Multi-Dimensional Scaling (MDS). Since this method uses all inter-beacon data between unlocated beacons, as well as between anchors and unlocated nodes, it performs better than iterative trilateration [15]. However, MDS-MAP requires all inter-beacon distance measurements and its performance depends on the quality of those measurements. For large localization areas it is difficult to move the mobile node in a path were all beacons are in range, therefore some inter-beacon distances must be estimated using the nearest path between those beacons. The number of hops used to estimate the inter-beacon measurements has a negative impact in the precision of such estimation. To avoid this problem, a modification proposed in [16], known as MDS-MAP (p), locates the beacons in local maps and later merges them in a global map containing all beacons. The basic idea is to use local maps with a limited number of hops. A modification of the MDS-MAP (p), known as LaMSM [17], further restricts the local maps to beacons that are fully connected. In this case no distance estimation by nearest path is necessary.

\section{Algorithm analysis}

In order to evaluate the performance of the proposed method, an LPS was simulated using MATLAB. The simulation is also used to choose two components of the algorithm not fully defined in Section 4: the mobile node path and the $2 \mathrm{D}$ localization algorithm used to locate the beacons once the inter-beacon distances are obtained. In the following simulations, unless stated different, the ranging data was generated with an additive Gaussian noise with zero mean and a standard deviation of $1 \mathrm{~cm}$. This standard deviation was chosen based on the precision typically obtained with ultrasonic LPSs [19]. All simulations were performed 100 times.

\subsection{Mobile node path analysis}

The basic conditions for the mobile node path were presented in Section 3: at least six noncollinear measurement points on a plane. To avoid aligned points, a circular path is used plus a measurement point at its center. The best parameters of such path: path radius $r$, the number of virtual nodes $n$, and the height $h$ from the nodes plane to the beacons plane are evaluated using the node configuration shown in Fig. 4. The default values used for these parameters are: $r=\frac{1.5}{\sqrt{2}} \mathrm{~m}, n=12$ and $h=2 \mathrm{~m}$. In all these tests three different beacon distributions $\left[N_{1}, N_{2}, N_{3}^{\prime}\right],\left[N_{1}, N_{2}, N_{3}, N_{4}\right]$, and $\left[N_{1}, N_{2}, N_{3}, N_{4}, N_{5}\right]$ are simulated.

In Fig. 5a we evaluate the inter-beacon distance mean error with different path radii. Since the error on the matrices of the linearized equations increase with the range distance, it was expected that the best radius value will be the one that minimized the distances from each virtual node to all beacons. However, the simulations show that the best path is the one that encloses the beacon nodes even with a radius much bigger than the inter-beacon distances. We observe that for very small radius the mobile node positions are too close and not much information is ob- 
tained with the measurements (as if we were measuring from only one mobile position). As the radius increases more information is obtained (the farther the points the more information we obtain). However, (9) shows that as the distance between beacons and the mobile increases the precision of the solution diminishes. So a mobile trajectory with big radius that does not place the mobile too far from the beacons is ideal. As observed, for this particular configuration, increasing the radius over $3 \mathrm{~m}$ also increases slowly the distance estimation error.

Another condition evaluated in this section is the number of virtual nodes used in the path. As seen in Fig. 5b the estimation of the inter-beacons distances improves with more redundant information obtained by more virtual nodes. However, it seems that the improvement obtained per added node decreases noticeably for more than 15 virtual nodes.

We finally evaluated the distance (height) between the beacons plane and the mobile path plane. As expected, the closer the virtual nodes get to the beacons the better is the solution. In this case, the distance between beacons and mobile plane will be limited by the emission/reception pattern of the nodes, since it is required that all points are visible to all beacons.

\section{2. $2 D$ localization algorithm analysis}

To evaluate the localization methods aforementioned in Section 4.2 we simulate a LPS composed by 49 beacons deployed on an area of $9 \mathrm{~m} \times 9 \mathrm{~m}$ as shown in Fig. 6 . We use a circular path for each group of nine beacons to obtain the inter-beacon distances between nearby beacons. The path is composed of 12 virtual nodes and has a radius of $\frac{3}{\sqrt{2}} \mathrm{~m}$ in order to encircle the beacons in each group. We limit the measurement range of the mobile node $(4,5$ and $9 \mathrm{~m})$ to obtain a different number of inter-beacon distances. Our goal is to verify which method performs better regardless of the number of inter-beacon distances available.

In Fig. 7 the cumulative error of the estimated beacons' position is presented for three different range limited

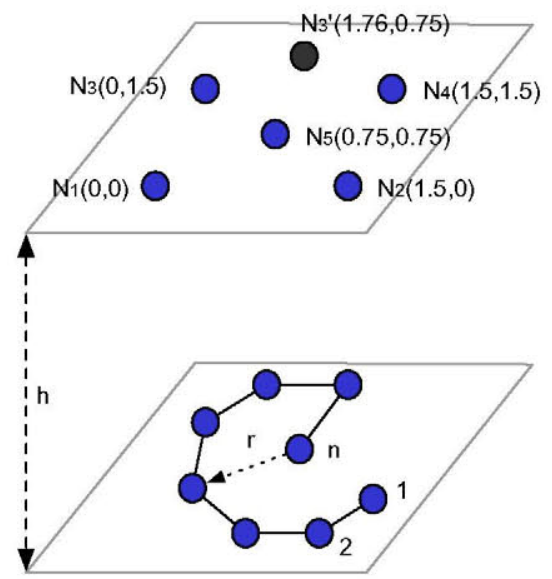

Fig. 4. Configuration used to evaluate the height $h$, radius $r$, and number of virtual nodes $n$ used in the mobile node path. Three beacon groups evaluated: $\left[N_{1}, N_{2}, N_{3}^{\prime}\right],\left[N_{1}, N_{2}, N_{3}, N_{4}\right]$, and $\left[N_{1}, N_{2}, N_{3}, N_{4}, N_{5}\right]$.

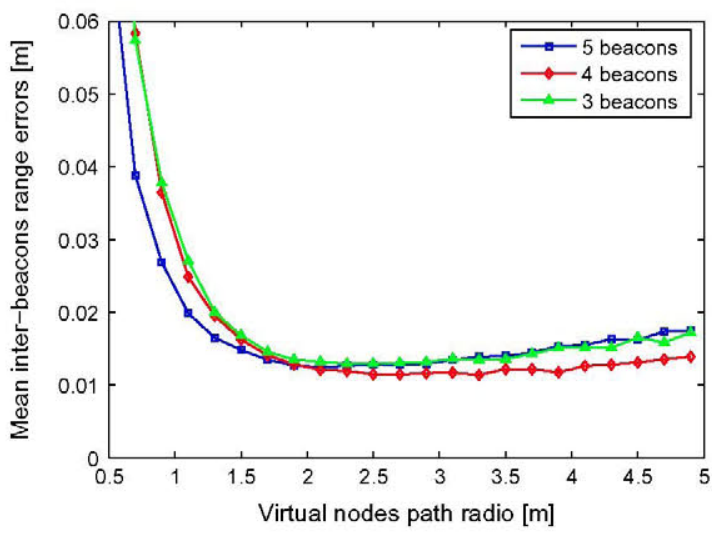

(a) Virtual nodes path radius

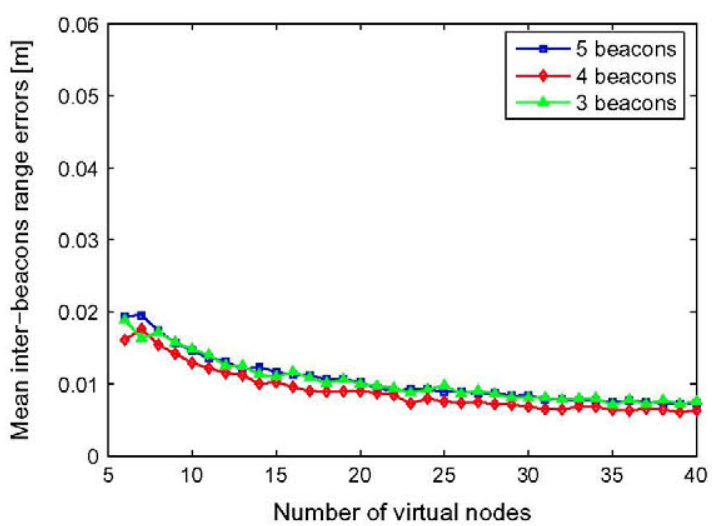

(b) Number of virtual nodes

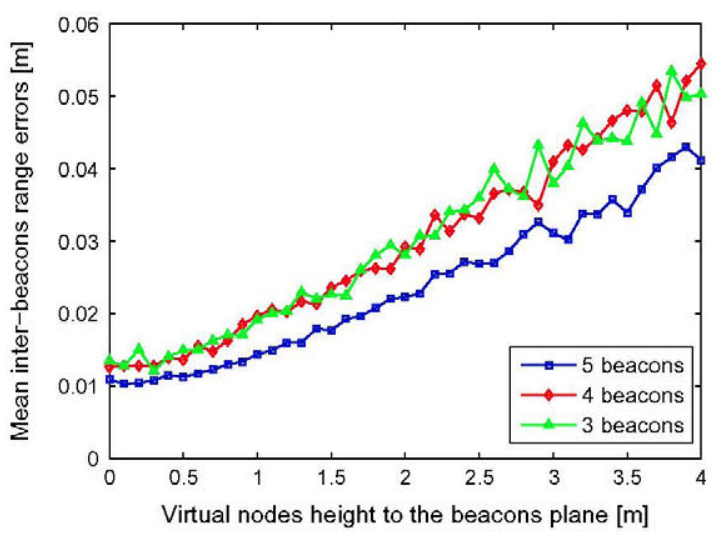

(c) Virtual nodes height

Fig. 5. Analysis of the inter-beacon distance error as a function of the mobile node path parameters and for three different beacon distributions.

measurements. In Fig. 7a the range limit simulated is $9 \mathrm{~m}$, therefore almost all inter-beacon distances are obtained. It can be seen that the trilateration method performs worst because of its accumulation of error. The performance of LaMSM and MDS-MAP (p) is almost the same, obtaining location errors under $4 \mathrm{~cm}$ on $90 \%$ of the beacons. MDS-MAP performs slightly worst than the other 
MDS methods obtaining location errors under $5 \mathrm{~cm}$ on $90 \%$ of the beacons. It seems that the lack of some inter-beacon distances, even a small number, affects the MDS estimation. This can be further observed in Fig. 7b where the limit range is $5 \mathrm{~m}$. In this case with less inter-beacon distances available, the trilateration algorithm performs better than the MDS-MAP (errors on $90 \%$ of the beacons under $12 \mathrm{~cm}$ for the trilateration method, $15.5 \mathrm{~cm}$ for MDS-MAP). Finally, in Fig. 7c a range limit of $4 \mathrm{~m}$ is simulated. In this case the MDS-MAP (p) still performs better than the MDSMAP, but not as good as the LaMSM method (errors on $90 \%$ of the beacons under $17 \mathrm{~cm}$ for MDS-MAP (p) and $13 \mathrm{~cm}$ for the LaMSM). The LaMSM method does decrease its performance compared with the previous simulations but it is still better than the other algorithms.

On Fig. 8 the cumulative error at $90 \%$ is shown for different localization areas keeping the distance between beacons constant at $1.5 \mathrm{~m}$. The simulated areas in $\mathrm{m}^{2}$ are: $[9,18,36,54,81,108]$ and the corresponding number of beacons are: $[9,15,25,35,49,63]$. The range limit used on this test is $5 \mathrm{~m}$.

As expected, for small areas all MDS based methods perform better than the trilateration. Since most inter-beacons distances are available, the MDS algorithms have a better distribution of the errors compared with the accumulative effect showed by the iterative trilateration. As the area increases, and more inter-beacon distances must be estimated by hops, the MDS-MAP rapidly decreases its performance. When only one hop is required for the beacons distance estimation, the MDS-MAP (p) performs exactly as the MDS-MAP. With large localization areas, the MDS-MAP (p) and the LaMSM performs better than the trilateration and the MDS-MAP, since the inter-beacon distances are never estimated using more than one hop. Based on the simulated tests the LaMSM is chosen to be used on the proposed auto-localization algorithm.

\section{Evaluation on an ultrasonic LPS}

In this section the linearized auto-localization method is compared with the inverse positioning method presented in [2]. The test is performed on the 3DLocus system [3], shown in Fig. 9, which is an acoustic LPS composed by

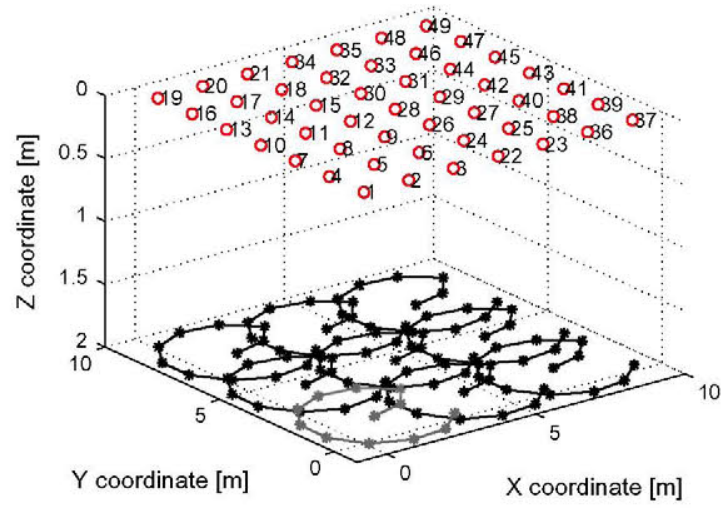

Fig. 6. Configuration used to evaluate the $2 \mathrm{D}$ localization algorithms, formed by 49 beacons deployed on an area of 9 by $9 \mathrm{~m}$. Each path is composed by 12 virtual nodes, one of them is shown in gray.

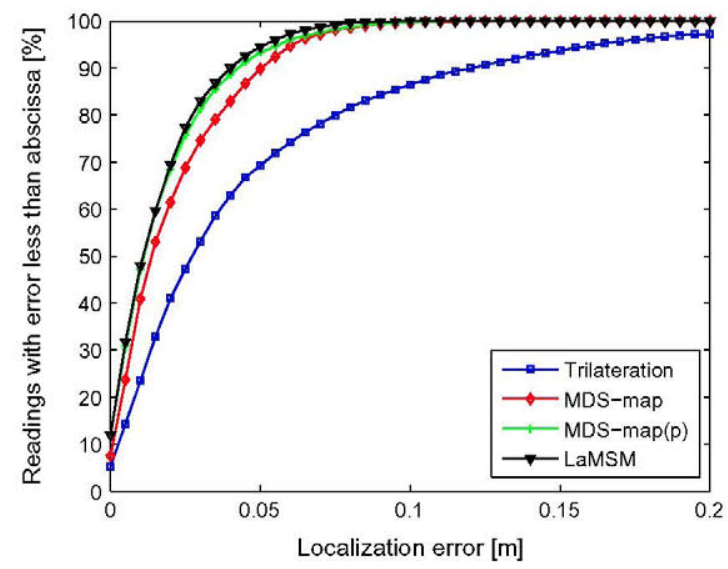

(a) Limit range: $9 \mathrm{~m}$

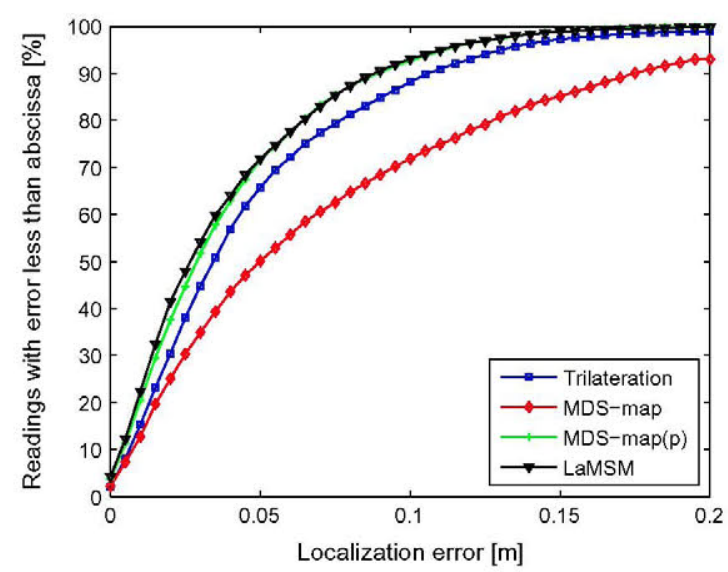

(b) Limit range: $5 \mathrm{~m}$

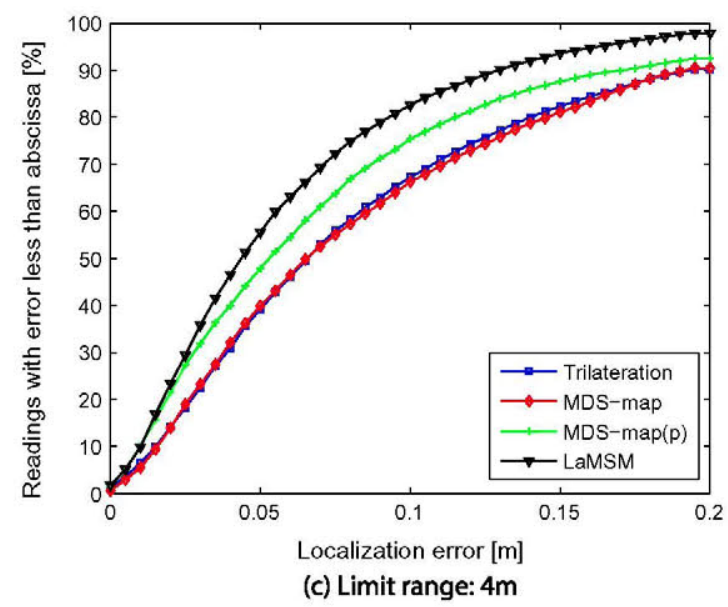

Fig. 7. Cumulative error of the estimated beacons' position by different 2D positioning algorithms.

seven beacons deployed on a cell of $2.8 \mathrm{~m} \times 2.8 \mathrm{~m} \times 2.8 \mathrm{~m}$. Since the inverse positioning method requires the exact position of the mobile node, a Staübli Unimation industrial robotic arm with a $50 \mu \mathrm{m}$ accuracy is used for positioning such node. 


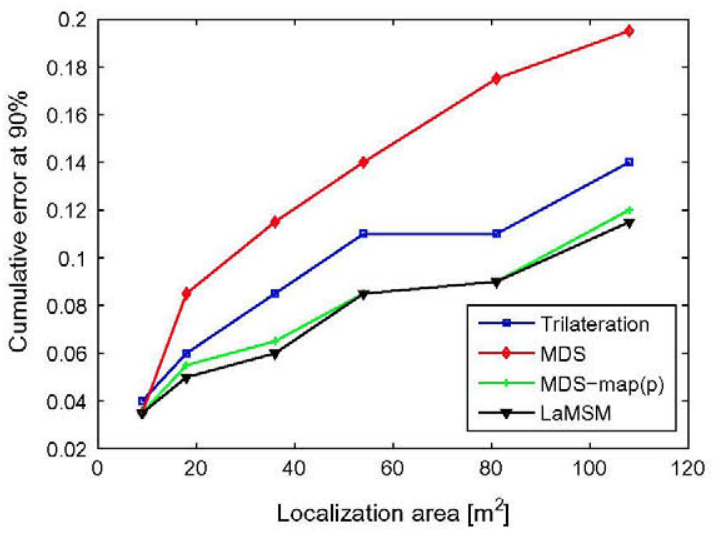

Fig. 8. Cumulative error at $90 \%$ as a function of the area covered by the beacon nodes for a measuring range of 5 meters. The simulated areas and corresponding number of beacons are: Areas in $m^{2}=[9,18,36,54,81$, $108]$ number of beacons $=[9,15,25,35,49,63]$.

A total of four measurement points were used to locate the beacons with the inverse positioning method. For the linearized auto-localization method a circular path of nine measurement points with unknown positions were used. For evaluation purposes, a total of one hundred measurements were made on each point. The distance measurements obtained with the 3DLocus presented a standard deviation of $0.23 \mathrm{~mm}$. The nodes' configuration used in the evaluation and the beacons' estimation obtained with the linearized auto-localization method and with the inverse positioning method is shown in Fig. 10.

In Fig. 11 the beacons' estimation obtained on several trials with the linearized auto-localization method its shown on the $X-Y$ plane. The $90 \%$ confidence ellipses presents a maximum variation of $1 \mathrm{~cm}$ on each axis between all estimates. Also, the estimated position of the beacons obtained with the inverse positioning method (used as reference) is shown. It can be seen that our method's solution

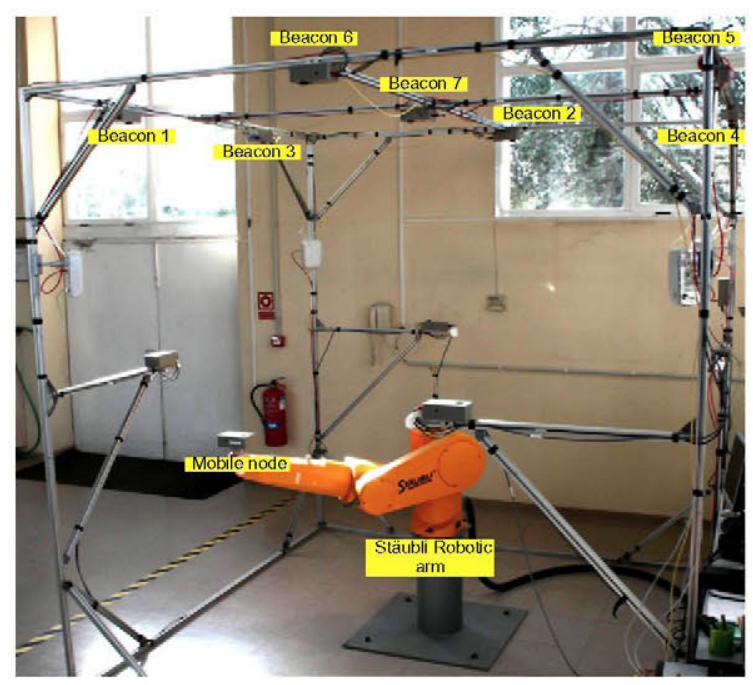

Fig. 9. 3Dlocus acoustic localization system.

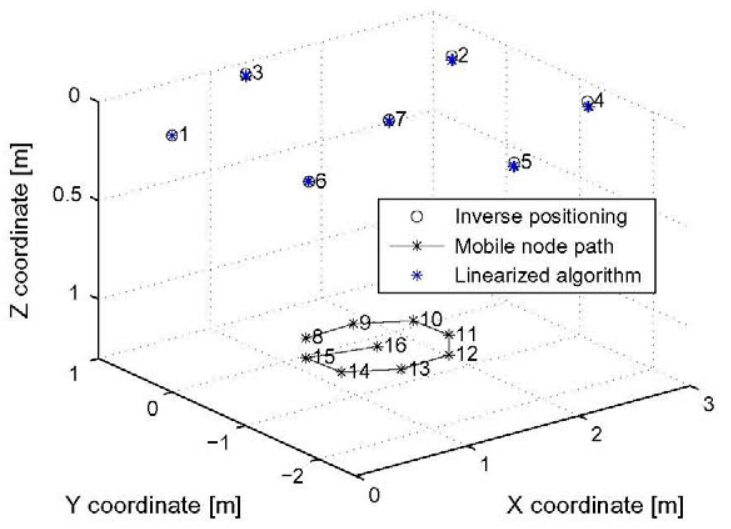

Fig. 10. 3Dlocus beacons positions obtained with the linearized algorithm and the inverse positioning method.

is close to the one obtained with the inverse positioning though there is a small offset between the estimated positions. This solution could be used as a first estimation of the beacons' position that later can be improved if necessary. Here, with an offset around $1.5 \mathrm{~cm}$, any optimization algorithm will easily find a more accurate solution.

Fig. 12 shows the average and maximum auto-localization difference, on each axis, of the linearized algorithm compared with the inverse positioning method. Since beacon one is used as origin no errors are present. On the other beacons the higher differences are seen on axis $Z$. Although the cell structure of the 3DLocus is positioned parallel to the floor, it seems that there is a small inclination that causes the altitude variation on the beacons. Since on the linearized method it is assumed that the beacons are on a plane parallel to the mobile path, any difference of altitude is reflected on $Z$ axis. On the other axes the average difference is below one centimeter, and the maximum obtained on all tests is below $1.5 \mathrm{~cm}$. The results indicate that the linearized method has a good accuracy in the autolocalization of beacon nodes.

\section{Conclusions}

In this paper a new solution for the auto -localization problem of 3D LPSs was presented based on the distance measurements between co-planar beacons nodes and a mobile one. Both, beacons and mobile locations, were unknown. While other techniques require an external positioning system (e.g., a second LPS or an odometric sensor) to estimate the positions of the mobile node, the proposed method uses only the measurements available on the LPS being located. The method is based on the linearization of the trilateration equations for three beacons by grouping nonlinear terms in additional variables. Since the linearized equations provide a closed-form solution, the method does not present local minima errors. By moving the mobile node on different paths the distance between more than three beacons can be obtained.

With the inter-beacon estimated distances all the LPS' beacons can be located using a 2D localization algorithm. Different localization methods were evaluated in order to 

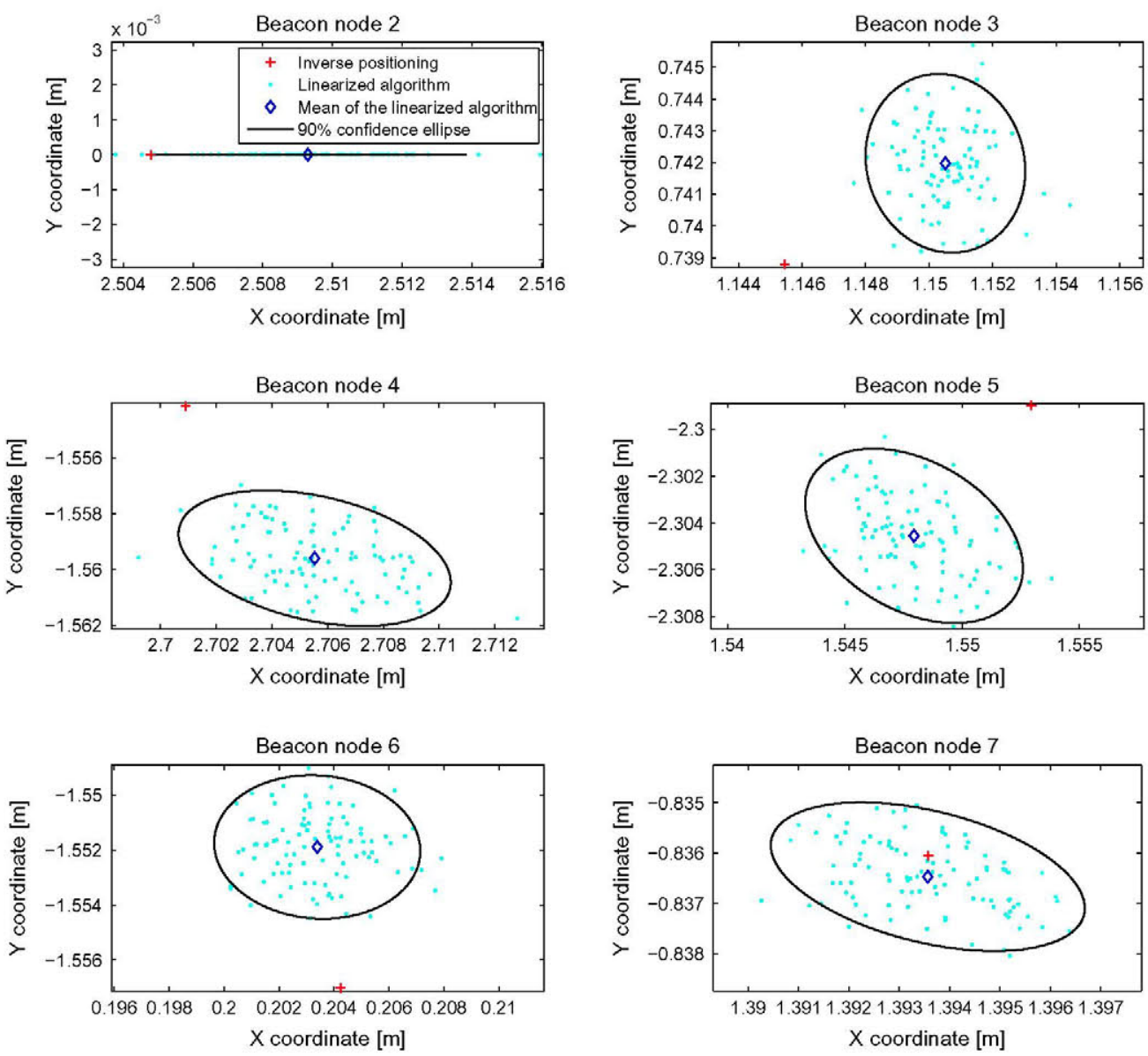

Fig. 11. $X-Y$ coordinates of 3Dlocus beacons' positions obtained with the linearized algorithm and the inverse positioning method.
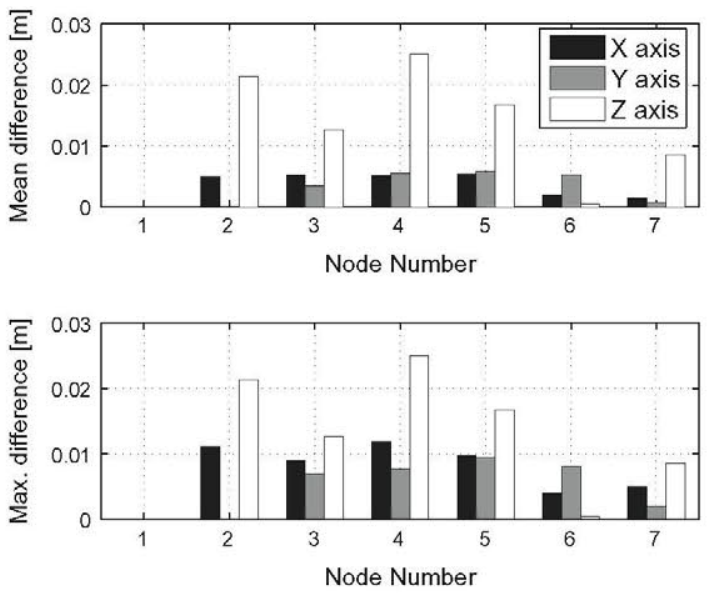

Fig. 12. Average and maximum position differences between the linearized algorithm and the inverse positioning method.

obtain the best solution with LPSs deployed on small and large areas. The simulations showed that the LaMSM method was best suited for large areas (where the inter-beacon distance estimations were few) and also on small areas (where most of the inter-beacon distances were available). Besides, the solutions obtained can be later improved by using them as initial conditions on other auto-localization techniques based on Bayesian or optimization methods.

Finally the method was evaluated on an acoustic LPS, the 3DLocus, and compared with the inverse positioning method. The results showed mean positioning differences below $1 \mathrm{~cm}$ on axes $X$ and $Y$ for each beacon. Higher differences are found in axis $Z$ due to the assumption that the beacons are on the same plane. The proposed method showed a good estimation of the beacons' position compared with one that requires a costly second positioning system, like the high-precision Staübli Unimation industrial robotic arm. The obtained results are very promising for a practical auto-calibration method that only requires to move a node around the localization area, without needing an initial estimation of the beacons' position or any external equipment.

Future work will focus on studying the effect that the node geometry has on the precision obtained by the linear equations. We expect an effect similar to the Geometric Dilution of Precision (GDOP) on GPS systems, though more complex since the measurement errors affect the matrix $A$ and $B$ of the $A X=B$ solution (in GPS the GDOP only takes into account the noise in $B$ ). 


\section{Acknowledgements}

The authors thank the financial support provided by projects LEMUR (TIN2009-14114-C04-03) and LAZARO (CSIC-PIE Ref.201150 E039).

\section{Appendix A. Linearization of the trilateration equations}

Our goal is to write an equation that includes the unknown inter-beacon distances $\left\{d_{12}, d_{13}, d_{23}\right\}$ and the measured distances $\left\{d_{14}, d_{24}, d_{34}\right\}$. Based on the LPS configuration of Fig. 2, we express the volume $V_{t}$ of the tetrahedron formed by the beacon nodes $\left\{N_{1}, N_{2}, N_{3}\right\}$ and the virtual node $\mathrm{N}_{4}$ using the Cayley-Menger determinant:

$288 V_{t}^{2}=\left|\begin{array}{ccccc}0 & 1 & 1 & 1 & 1 \\ 1 & 0 & d_{12}^{2} & d_{13}^{2} & d_{14}^{2} \\ 1 & d_{12}^{2} & 0 & d_{23}^{2} & d_{24}^{2} \\ 1 & d_{13}^{2} & d_{23}^{2} & 0 & d_{34}^{2} \\ 1 & d_{14}^{2} & d_{24}^{2} & d_{34}^{2} & 0\end{array}\right|$.

By expanding (A.1) and dividing all by $\frac{1}{2 d_{12}^{2}}$ we obtain:

$$
\begin{aligned}
144 \frac{V_{t}^{2}}{d_{12}^{2}}= & d_{13}^{2} d_{24}^{2}-d_{13}^{2} d_{23}^{2}-d_{34}^{4}+d_{14}^{2} d_{23}^{2}-d_{14}^{2} d_{24}^{2} \\
& -d_{12}^{2} d_{34}^{2}+d_{13}^{2} d_{34}^{2}+d_{14}^{2} d_{34}^{2}+d_{23}^{2} d_{34}^{2} \\
& +d_{24}^{2} d_{34}^{2}-\frac{d_{13}^{2} d_{24}^{4}}{d_{12}^{2}}-\frac{d_{13}^{4} d_{24}^{2}}{d_{12}^{2}}-\frac{d_{14}^{2} d_{23}^{4}}{d_{12}^{2}} \\
& -\frac{d_{14}^{4} d_{23}^{2}}{d_{12}^{2}}+\frac{d_{13}^{2} d_{14}^{2} d_{23}^{2}}{d_{12}^{2}}+\frac{d_{13}^{2} d_{14}^{2} d_{24}^{2}}{d_{12}^{2}} \\
& +\frac{d_{13}^{2} d_{23}^{2} d_{24}^{2}}{d_{12}^{2}}-\frac{d_{13}^{2} d_{14}^{2} d_{34}^{2}}{d_{12}^{2}}+\frac{d_{14}^{2} d_{23}^{2} d_{24}^{2}}{d_{12}^{2}} \\
& +\frac{d_{13}^{2} d_{24}^{2} d_{34}^{2}}{d_{12}^{2}}+\frac{d_{14}^{2} d_{23}^{2} d_{34}^{2}}{d_{12}^{2}}-\frac{d_{23}^{2} d_{24}^{2} d_{34}^{2}}{d_{12}^{2}}
\end{aligned}
$$

In order to linearize (A.2) we begin grouping all nonlinear terms of the unknown distances in additional variables:

$$
\begin{aligned}
& \left(d_{34}^{2} d_{12}^{2}-d_{34}^{2} d_{23}^{2}-d_{13}^{2} d_{34}^{2}\right)+\left(\frac{d_{14}^{2} d_{23}^{4}}{d_{12}^{2}}-d_{23}^{2} d_{14}^{2}-\frac{d_{13}^{2} d_{14}^{2} d_{23}^{2}}{d_{12}^{2}}\right) \\
& +\left(\frac{d_{13}^{4} d_{24}^{2}}{d_{12}^{2}}-\frac{d_{13}^{2} d_{23}^{2} d_{24}^{2}}{d_{12}^{2}}-d_{13}^{2} d_{24}^{2}\right)+\left(d_{13}^{2} d_{23}^{2}+144 \frac{V_{t}^{2}}{d_{12}^{2}}\right) \\
& -\left(\frac{d_{14}^{2} d_{23}^{2} d_{24}^{2}}{d_{12}^{2}}+\frac{d_{23}^{2} d_{14}^{2} d_{34}^{2}}{d_{12}^{2}}-\frac{d_{23}^{2} d_{22}^{2} d_{34}^{2}}{d_{12}^{2}}-\frac{d_{14}^{4} d_{23}^{2}}{d_{12}^{2}}\right) \\
& -\left(\frac{d_{13}^{2} d_{34}^{2} d_{24}^{2}}{d_{12}^{2}}-\frac{d_{13}^{2} d_{14}^{2} d_{34}^{2}}{d_{12}^{2}}+\frac{d_{13}^{2} d_{13}^{2} d_{24}^{2}}{d_{12}^{2}}-\frac{d_{13}^{2} d_{24}^{4}}{d_{12}^{2}}\right)+D_{314} D_{324}=0, \text { (A.3) }
\end{aligned}
$$

where $D_{314} D_{324}=\left(d_{34}^{2}-d_{14}^{2}\right)\left(d_{34}^{2}-d_{24}^{2}\right)$.

We define the new unknown variables $X_{i}$ of the linearized equation, where each variable corresponds with the ith element of matrix $X$ defined in (4):

$$
\begin{aligned}
& -X_{1} d_{34}^{2}-X_{3} d_{14}^{2}-X_{2} d_{24}^{2}+X_{6}+D_{314} D_{324} \\
& -\left(d_{14}^{2} d_{24}^{2}+d_{34}^{2} d_{14}^{2}-d_{34}^{2} d_{24}^{2}-d_{14}^{4}\right) X_{5} \\
& -\left(d_{34}^{2} d_{24}^{2}-d_{34}^{2} d_{14}^{2}+d_{14}^{2} d_{24}^{2}-d_{24}^{4}\right) X_{4}=0
\end{aligned}
$$

Using (3) we obtain the linearized trilateration equations:

$X_{1} d_{34}^{2}+X_{2} d_{24}^{2}+X_{3} d_{14}^{2}+D_{314} D_{214} X_{4}-D_{324} D_{214} X_{5}-X_{6}=D_{314} D_{324}$

We can apply (A.5) (obtained for the virtual node $k=4$ ) to the remaining virtual nodes $k=5, \ldots, 9$ in order to obtain the linearized trilateration equations. These equations are presented in matrix form in Section 3.1 .

In order to obtain the final positions of the beacons $\left\{N_{1}\right.$, $N_{2}, N_{3}$ \} from the solution obtained in (4), based on the LPS configuration of Fig. 2, we use the inter-beacon distance equations:

$d_{12}^{2}=N_{2 x}^{2}$,

$d_{13}^{2}=N_{3 x}^{2}+N_{3 y}^{2}$,

$d_{23}^{2}=\left(N_{3 x}-N_{2 x}\right)^{2}+N_{3 y}^{2}$.

Expanding (A.8) and using (A.6) and (A.7) we obtain:

$$
\begin{aligned}
d_{23}^{2} & =N_{3 x}^{2}+N_{2 x}^{2}-2 N_{2 x} N_{3 x}+N_{3 y}^{2} \\
& =d_{13}^{2}+d_{12}^{2}-2 N_{2 x} N_{3 x}=d_{13}^{2}+d_{12}^{2}-2 d_{12} N_{3 x}
\end{aligned}
$$

Using (A.9) we obtain an expression for $N_{3 x}$ :

$N_{3 x}=\frac{d_{13}^{2}+d_{12}^{2}-d_{23}^{2}}{2 d_{12}}$,

and from (A.7) we obtain $N_{3 y}$ :

$N_{3 y}=\sqrt{d_{13}^{2}-N_{3 x}^{2}}$.

From A.6, A.10 and A.11 we obtain the inter-beacon coordinates expressed in (7).

\section{References}

[1] J. Hightower, G. Borriello, Location systems for ubiquitous computing, Computer 34 (2001) 57-66.

[2] A. Mahajan, F. Figueroa, An automatic self-installation and calibration method for a $3 \mathrm{D}$ position sensing system using ultrasonics, Robotics and Autonomous Systems 28 (1999) 281-294.

[3] J. Prieto, A. Jimenez, J. Guevara, J. Ealo, F. Seco, J. Roa, F. Ramos, Performance evaluation of 3D-locus advanced acoustic LPS, IEEE Transactions on Instrumentation and Measurement (2009) 23852395.

[4] D. Ruiz, J. Ureña, J.C. García, A. Jiménez, Â. Hernández, J.J. García, LPS auto-calibration algorithm with predetermination of optimal zones, Sensors (2011) 10398-10414.

[5] A. Nishitani, Y. Nishida, T. Hori, H. Mizoguchi, Portable ultrasonic 3D tag system based on a quick calibration method, in: IEEF International Conference on Systems, Man and Cybernetics, The Hague, The Netherlands, 2004, pp. 1561-1568.

[6] P. Duff, H. Muller, Autocalibration algorithm for ultrasonic location systems, in: ISWC '03: Proceedings of the 7th IEEE International Symposium on Wearable Computers, IEEE, Washington, DC, USA, 2003, pp. 62-68.

[7] E. Olson, J. Leonard, S. Teller, Robust range-only beacon localization, in: Proceedings of Autonomous Underwater Vehicles 2004, IEEE/OES Maine, USA, 2004, pp. 66-75.

[8] D. Ruiz, J. Ureña, J.C. Garćia, Á. Hernández, E. García, J. Aparicio, Simultaneous mobile robot positioning and LPS self-calibration in a smart space, Proceedings of the 2010 IEEE International Symposium on Industrial Electronics, IEEE, Bari, Italy, 2010, pp. 2865-2870.

[9] R. Mautz, W. Ochieng, A robust indoor positioning and autolocalisation algorithm, Journal of Global Positioning Systems (2007) 38-46.

[10] N.B. Priyantha, H. Balakrishnan, E. Demaine, S. Teller, Mobileassisted localization in wireless sensor networks, in: INFOCOM 
2005. 24th Annual Joint Conference of the IEEE Computer and Communications Societies, IEEE, Miami, USA, pp. 172-183.

[11] J. Guevara, A.R. Jiménez, A.S. Morse, J. Fang, J.C. Prieto, F. Seco, Autolocalization in local positioning systems: a closed-form range-only solution, in: Proceedings of the 2010 IEEE International Symposium on Industrial Electronics, IEEE, Bari, Italy, 2010, pp. 2834-2840.

[12] W. Kuo, V. Uppuluri, A review of error propagation analysis in systems, Microelectronics Reliability (1983) 235-248.

[13] H. Antia, Numerical Methods for Scientists and Engineers, Tata McGraw-Hill, New Delhi, India, 1995.

[14] A. Savvides, C.-C. Han, M.B. Strivastava, Dynamic fine-grained localization in ad-hoc networks of sensors, in: Proceedings of the 7 th annual international conference on mobile computing and networking, MobiCom 2001, ACM, New York, USA, 2001, pp. 166179.

[15] A. Youssef, M. Youssef, A taxonomy of localization schemes for wireless sensor networks, in: The 2007 International Conference on Wireless Networks, ICWN, Nevada, US, 2007, pp. 444-450.

[16] Y. Shang, Improved Mds-based localization, in: INFOCOM 2004. 24th Annual Joint Conference of the IEEE Computer and Communications Societies, IEEE, Hong Kong, China, 2004, pp. 2640-2651.

[17] E. Kim, S. Woo, C. Kim, K. Kim, LaMSM: localization algorithm with merging segmented maps for underwater sensor networks, in: Proceedings of the 2007 Conference on Emerging Direction in Embedded and Ubiquitous Computing, EUC 2007, Springer-Verlag, Berlin, Germany, 2007, pp. 445-454.

[18] A.C. Davison, Statistical Models, Cambridge University Press, New Cambridge, UK, 2003.

[19] R. Mautz, The challenges of indoor environments and specification on some alternative positioning systems, in: 6th Workshop on Positioning, Navigation and Communication, WPNC 2009, IEEE, Hannover, Germany, 2009, pp. 29-36.

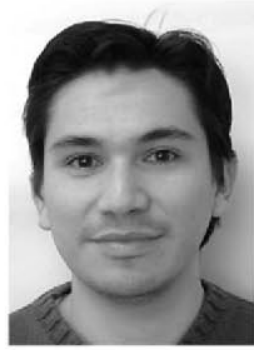

systems.
Jorge Guevara was born in Lima, Peru, in 1978 . $\mathrm{He}$ received the B.S. degree in electronics engineering from the Universidad Católica Nuestra Señora de la Asunción, Asunción, Paraguay, in 2004. He is currently working toward the Ph.D. degree in electric engineering with the Centro de Automática y Robótica, Consejo Superior de Investigaciones Científicas (CSIC) - UPM, Madrid, Spain.

His research interests are in the area of localization systems, in particular, automatic calibration methods for ultrasonic positioning

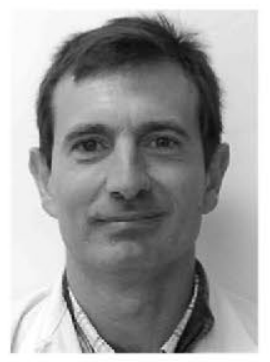

Antonio Ramón Jimênez Ruiz received the degree in Physics and Computer Science and the Ph.D. degree in Physics from the Universidad Complutense de Madrid, Spain, in 1991 and 1998, respectively. From 1991 to 1993, he worked in industrial laser applications with the Technological Center of Madrid (CETEMA). Since 1994, he has been a researcher within the Instituto de Automática Industrial - IAI (now named Centro de Automática y Robótica - CAR), belonging both to the largest Public-funded Agency for Research in Spain (Consejo Superior de Investigaciones Científicas-CSIC). Since 2005 , he belongs to the LOPSI group, specialized in designing local positioning solutions for indoor localization and navigation. His current research interests include: Sensors, Signal Processing and Algorithms applied to the precise indoor/outdoor Localization of persons and robots. He has a special interest in creating prototypes to demonstrate the functionality of Local Positioning Systems (LPSs) using acoustic/ ultrasonic and RFID technology, as well as, Pedestrian Dead-Reckoning (PDR) solutions using foot-mounted IMUs. He is author and reviewer of many international journals and communications in the field.

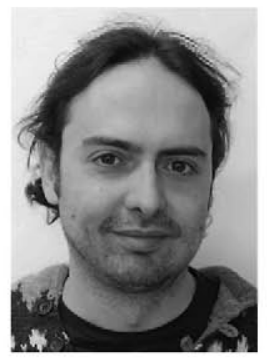

José Carlos Prieto was born in León, Spain, in 1978. He received the Technical degree in industrial electronics and the B.Sc. degree in electronics engineering from the Universidad de Extremadura, Badajoz, Spain, in 1999 and 2003, respectively, and the Master's degree in robotics from the Universidad Politécnica de Madrid, Madrid, Spain, in 2007. He is currently working toward the Doctoral degree in robotics with the Universidad de Alcalá, Madrid. Since 2004, he has been a Researcher with the Centro de Automática y Robótica, Consejo Superior de Investigaciones Científicas (CSIC)-UPM, Madrid.

His research interests are focused in localization systems, mainly those based in ultrasonic signals, with special emphasis in signal design and processing, positioning algorithms, robustness, standardization, optimal configurations, calibration methods, and development of new transducers.

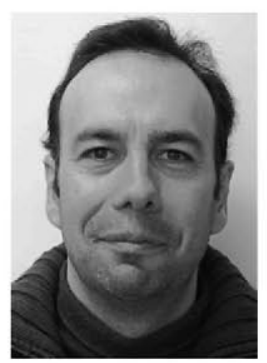

Fernando Seco was born in Madrid, Spain, in 1972. He received the degree in physics from the Universidad Complutense of Madrid, Madrid, in 1996 and the Ph.D. degree in physics from the Universidad Nacional de Educación a Distancia (UNED), Madrid, in 2002. His dissertation dealt with the generation of ultrasonic waves applied to a magnetostrictive linear position sensor. Since 1997. he has been with the Centro de Automática $y$ Robótica, Consejo Superior de Investigaciones Cientificas (CSIC)-UPM, Madrid, where he holds a research position.

His main research interest lies in the design and development of Local Positioning Systems (LPSs), particularly those based on ultrasound and RFID, and specifically on the topics of signal processing of ultrasonic signals, multilateration algorithms, and Bayesian localization methods. 